\title{
IMPLEMENTACIÓN DE UN SISTEMA DE INTERNET DE LAS COSAS PARA OPTIMIZAR LA GESTIÓN DEL AGUA EN LA AGRICULTURA DE LA REGIÓN TACNA
}

IMPLEMENTATION OF AN INTERNET OF THINGS SYSTEM TO OPTIMISE WATER MANAGEMENT IN AGRICULTURE IN THE TACNA REGION

Christian CÉsar ACERo CATACORA 1

(D) https://orcid.org/ 0000-0002-8675-8036

ENRIQUE LANCHIPA VALENCIA ${ }^{2}$

(D) https://orcid.org/ 0000-0001-8164-9781

Información del artículo: Recibido: 21/01/2021 Aceptado: 01/06/2021

Publicado: 28/06/2021

\footnotetext{
${ }^{1}$ Escuela de Ingeniería Sistemas, Universidad Privada de Tacna

${ }^{2}$ Docente en la Escuela de Ingeniería Sistemas, Universidad Privada de Tacna E-mail: ${ }^{1}$ christian.acero@masterprojectperu.com, ${ }^{2}$ elanchipa@upt.pe
} 


\section{Resumen}

El diseño óptimo de un sistema autónomo para la gestión del recurso hídrico del agua en la agricultura es consecuencia de un estudio detallado sobre las condiciones en el cual funcionará el sistema, de acuerdo con los rigores de calidad. En ese contexto se aplica el concepto de Internet de las Cosas en conjunto con un Sistema de Control, para la optimización del proceso de regadío tradicional, optimización de la gestión del agua, mejora del producto final y ahorro de energía y tiempo.

La suma de esfuerzos para realizar un sistema autónomo controlado y monitoreado desde Internet, se define actualmente como Smart Farming o Agricultura Inteligente, es la mezcla de tecnologías informáticas y dispositivos capaces de interrelacionarse entre sí, para ofrecer al usuario el control, autonomía y análisis de datos en tiempo real para la toma de decisiones. En tal sentido, para la comprobación de su optimización de la gestión del agua se ha establecido la comparación de un sistema de regadío con Internet de las Cosas y un sistema de regadío tradicional manual.

En un terreno de cultivo tecnificado, para regar por el método de goteo, el cual es activado por el agricultor o se programa el tiempo de un dispositivo para que se riegue entre diferentes escalas de tiempo. El terreno también cuenta con un reservorio de 600000 litros de agua para $3535 \mathrm{~m}^{2}$ de área de cultivo.

Los resultados indicaron que la implementación de un Sistemas de Internet de las Cosas, denominado Damla, optimizó el uso del recurso hídrico hasta un 21.25 \% en el riego del cultivo e indirectamente el ahorro de energía por el uso de las bombas de agua.

Palabras clave: Internet de las Cosas; control de procesos; agricultura inteligente.

\section{Abstract}

The optimal design of an autonomous system for water resource management in agriculture is a consequence of a detailed study of the conditions under which the system will operate, according to the rigours of quality. In this context, the concept of the Internet of Things is applied in conjunction with a Control System for the optimisation of the traditional irrigation process, optimisation of water management, improvement of the final product and savings in energy and time.

The sum of efforts to realise an autonomous system controlled and monitored from the Internet, is currently defined as Smart Farming or Intelligent Agriculture, it is the mixture of computer technologies and devices capable of interrelating with each other, to offer the user control, autonomy and data analysis in real time for decision making. In this sense, a comparison of an irrigation system with the Internet of Things and a traditional manual irrigation system has been established to verify the optimisation of water management.

In a technified cultivation plot, to irrigate by the drip method, which is activated by the farmer or the time of a device is programmed to irrigate between different time scales. The site also has a reservoir of 600000 litres of water for $3535 \mathrm{~m}^{2}$ of cultivation area.

The results indicated that the implementation of an Internet of Things system, called Damla, optimised the use of water resources by up to $21.25 \%$ in irrigating the crop and indirectly saved energy by using water pumps.

Keywords: Internet of Things; process control; smart farming. 


\section{Introducción}

Los avances tecnológicos recientes están permitiendo el desarrollo y lanzamiento de productos electrónicos cada vez más pequeños y sofisticados de bajo costo. Actualmente estas tecnologías se utilizan en una variedad de sectores; la agricultura es seguramente uno de ellos. Por siglos la agricultura viene desempeñando un papel importante en la economía de cada cultura, país y nación. Una sus mejoras hace algunas décadas es agricultura de precisión (AP). El objetivo principal de la agricultura de precisión es, mediante la recolección de datos del medio ambiente o microclima, mejorar la calidad de los productos y mantener una agricultura sostenible. Para lograrlo, se quiere de optimizar los recursos utilizados en los procesos agrícolas, principalmente en el sistema de riego. Entonces el agua juega un papel crucial en el ciclo de vida de las plantas y la de todos los seres vivos. Por ello, el agua proporcionada por la precipitación natural, como las lluvias, no proporcionan la cantidad de agua que las plantas requieren para crecer de manera saludable. Al ser un recurso escaso, se determinó que al menos el $3 \%$ del agua del mundo es dulce, siendo el agua de mar restante y no potable. El $2.5 \%$ de este $3 \%$ está congelado, es decir, la humanidad debe confiar en el $0.5 \%$ del agua restante, tal como indican el informe de Fry (2005). La agricultura consume alrededor del $70 \%$ del agua dulce. Este porcentaje puede disminuirse realizando una gestión eficiente del agua cuando se trata de riego. Según Yule, Hedley \& Bradbury (2009), es importante plantear la optimización según:

- Calidad del cultivo, con la cantidad exacta de agua que necesitan, no solo ahorraría agua, sino que también mejoraría la calidad del cultivo.

- Económicos, el costo del agua seguirá creciendo junto con la disminución de la disponibilidad de agua dulce. Al proporcionar soluciones para que el cultivo reciba la cantidad correcta de agua, se puede ahorrar dinero. El rendimiento también se puede aumentar, y con ello, su beneficio comercial.

- El ahorro del agua se busca poder regar las plantas de forma más selectiva y precisa de acuerdo con las necesidades individuales de las plantas.

De la misma manera, Kamienski, Soininen \& otros (2019), manifiestan que una gestión inteligente del agua para el riego de precisión es esencial para incrementar la calidad del cultivo y disminuir costos, mientras que contribuye a la sostenibilidad ambiental. Es por ello que el uso de Internet de las Cosas es la opción natural para las aplicaciones inteligentes para la gestión del agua. Ahamed (2019), manifiesta que los sistemas basados en Internet de las cosas proporcionan soluciones a los agricultores de gran escala. También manifiesta que gracias a la lectura de los datos recolectados por lo sensores pueden ser gran utilidad para los profesionales de la agricultura, ya que sirven para dar un seguimiento a tiempo real del cultivo. Por ello, la implementación óptima de un sistema de Internet de las Cosas debe operar de manera autónoma y efectiva, con datos que son procesados a tiempo real para un análisis temprano de decisiones y el funcionamiento efectivo del proceso, esto se traduce en un ahorro de tiempo para operador o agricultor, mejora del cultivo y ahorro económico.

Según el Instituto Internacional de Gestión del Agua, la agricultura, que consume alrededor del 70\% de las extracciones de agua dependiendo del tipo de país, compite constantemente con los usos domésticos, industriales y ambientales por un suministro de agua escaso. Para 2025, la población mundial probablemente aumentará a 7.9 billones, de los cuales más del $80 \%$ vive en países en desarrollo y el $58 \%$ en áreas urbanas de rápidocrecimiento, según las tendencias dietéticas esto se traducirá en una demanda de agua aún mayor si los alimentos producidos son para proporcionar una nutrición adecuada. En un intento de solucionar este problema cada vez mayor, muchas organizaciones mundiales están intentado formar métodos más efectivos de gestión del agua. (Mark, Cai, \& A. Cline, 2002). Es por eso que los gobiernos y organizaciones a nivel mundial están buscando soluciones innovadoras para tener una mejor gestión de sus recursos naturales, tales como el agua. El estrés hídrico, es decir, la disminución de la cantidad de agua consumible se está expandiendo a nivel mundial a un ritmo alarmante, de acuerdo con la ONU. Siendo la escasez de agua quien afecta a más del $40 \%$ de la población mundial, una cifra alarmante que probablemente crecerá con el aumento de las 
temperaturas globales producto del cambio climático y el crecimiento de la población.

Perú se encuentra entre los 30 países que enfrentan el estrés hídrico y la escasez más severos a nivel mundial. Siendo la agricultura el principal usuario de agua en nuestra sociedad, utilizando el $80 \%$ de los retiros totales para irrigar un área de 128 '521,560 ha., solo en la región costa se encuentra un $11.6 \%$, el cual tiene una mayor probabilidad de sufrir sequias. El uso doméstico, que tiene la prioridad de asignación según la ley, del $12.2 \%$ del agua. Pero el $24 \%$ de los peruanos no tiene acceso a los servicios de agua, el $43 \%$ no tiene instalaciones de saneamiento básico y solo el $22 \%$ del agua residual doméstica es tratada adecuadamente. La minería y la industria consumen una proporción menor de la demanda nacional de agua y ambos sectores producen un valor económico significativamente más alto que los demás. Aunque el sector minero utiliza solo el $2 \%$ del total de extracciones de agua (Alegria, 2013). Además, las áreas de glaciares $\left(2,600 \mathrm{Km}^{2}\right)$ se han reducido en un $22 \%$ en los últimos 27 a 35 años, los niveles de agua en las lagunas naturales y represas están descendiendo a falta de lluvia en la sierra, un gobierno que no gestiona bien el recurso hídrico, minería ilegal, descuido humano en empresas petroleras están acabando con este recurso tan valioso, por ende, se ven afectados la agricultura, ganadería y comunidad en general.

En Tacna, las partes interesadas en el agua actúan de manera independiente y competitiva, ya que algunas veces parecen consumir el agua de manera beligerante porque no están seguras de "lo que ganan al conservar". Si bien muchos estudios han relacionado la escasez de agua con todo, desde el cambio climático hasta las demandas de agua en la minería, o la falta de educación sobre sostenibilidad, el caso particular de Tacna experimenta todas estas cosas. En otros lugares, la adaptación ha sido eficiente y efectiva en respuesta al empeoramiento de la disponibilidad de agua, pero en Tacna, se ha trabajado poco para la creación de soluciones innovadoras aplicables (Conor, 2012). En la Región de Tacna la falta de optimización de los recursos hídricos en la agricultura es baja, pese a los años han pasado y los gobiernos cambiando, solo se ha logrado un sistema de riego tradicionales. Está es una oportunidad para el uso de tecnologías que permitan al agricultor gestionar de manera más efectiva un recurso tan valioso como el agua, evitando que más gente siga haciendo perforaciones de pozos ilegales que sigan agotando el acuífero que tenemos.

"El manejo del agua de riego tiene que adaptarse a los crecientes requerimientos de alimentos (...). Las principales tecnologías que se utilizarán en los países en desarrollo con abundante mano de obra y escaso capital son el riego por goteo y subterráneo, que depende de pequeñas cantidades frecuentes de agua directamente a la zona de raíces de los cultivos. La agricultura siempre seguirá siendo el usuario más dominante de los recursos hídricos a nivel mundial y está destinada a aumentar especialmente en las regiones áridas y semiáridas" (Luquet, Vidal, Smith, \& Dauzat, 2005).

Por ello resulta necesario implementar esta solución para evaluar el impacto al aplicar tecnologías de información para optimizar la gestión del agua en la agricultura, evaluando costobeneficio de la propuesta de valor. Además, los sistemas de información orientados a las exigencias del futuro tecnológico en la agricultura deben optimizar los recursos tales como el agua, calidad de tierra y energía ya que si no se implementan traerían consecuencias negativas para el agricultor a mediano y largo plazo para las áreas de cultivo, en especial en zonas áridas como la costa sur del Perú. Este estudio es un aporte a la optimización el recurso hídrico en la agricultura de la Región Tacna a través de la implementación de sistema loT (Internet of Things), que está basado en un dispositivo propio, denominado Damla, que es un dispositivo embebido (compuesto de sensores y actuadores), también una aplicación web y una aplicación servidor para la comunicación entre Damla y la aplicación web. Así, en conjunto cumplan con el concepto de Internet of Things orientado a la agricultura. Permitiendo en basea diferentes sensores determinar el tiempo de riego óptimo, accionar válvulas y bomba de agua de manera autónoma. A lado del agricultor, la aplicación le permitirá visualizar el nivel de humedad del suelo, humedad relativa aire, temperatura ambiente, estados de motores y válvulas, adicionalmente permitirá visualizar nivel de radiación UV y calidad del aire para que el agricultor pueda utilizar los datos para la toma de decisiones sobre el cultivo. Para que cumpla con el concepto Internet of Things, todos los datos podrán ser visualizados desde cualquier parte del mundo ya que los dispositivos estarán conectados a Internet, en caso el usuario desee hacer una operación de manera 
remota, podrá hacerlo desde una app web.

De esta manera se busca que el agricultor pueda gestionar sus terrenos de manera remota, optimizar el recurso hídrico a través de la lectura y procesamiento de datos de los sensores. A nivel del Perú empresas internacionales están ingresando al mercado proponiendo soluciones similares, pero a precios descomunales y poco accesibles para el agricultor. Es por ello que se plantea la implementación de un sistema de Internet of Things robusto que procese los datos de los sensores a tiempo real y sean visualizados a través de un $\mathrm{HMI}$ en diferentes dispositivos y lograr que sea accesible para los agricultores.

Dias Sales (2015); de Lisboa, Portugal; concluye que, a través del estudio e investigación de la literatura relacionada con los estudios de campo relacionados a Internet de las cosas, Maquina-aMaquina (Machine to machine) y de las redes inalámbricas de sensores y actuadores, concluye que esta integración podría aplicarse a una gran cantidad de casos de uso. Sekharm, Manujunatha \& Sundeep (2013); sostienen que la implementación de sensores, redes inalámbricas y controladores ANN para un sistema automático de riego tienden a obtener un ahorro de energía y agua, y al utilizar el concepto ANN, obtuvieron resultados precisos al calcular la humedad del suelo requerida a partir de los parámetros de entrada. Para Villabos, Orgaz, Testi, \& Fereres (2000); que las mediciones de la evaporación por encima y por debajo de un huerto de olivos y la conductancia aerodinámica permitió la calibración de un modelo de transpiración de olivos. El modelo se combinó con un modelo de evaporación del suelo y se contrastó con un conjunto de datos independientes, lo que indica un rendimiento justo a menos que una fracción sustancial de la superficie del suelo sea mojada por los emisores de riego, lo que el modelo no toma en cuenta. El coeficiente de cultivo promedio es bastante bajo debido a la baja cobertura del suelo y al control mejorado de la conductancia del dosel por las respuestas estomáticas al VPD. Estos resultados indican que las estimaciones de los requerimientos de agua de aceituna no pueden evaluarse con precisión con el método del coeficiente de cultivo, enfoque, como el que se presenta aquí es necesario.

Muñoz, Calero \& Marín (2013), al hacer uso de un sistema de riego inteligente se obtiene un significativo ahorro de los recursos hídricos frente al sistema de riego tradicional, manteniendo el mismo nivel de producción como se demuestra en la sección seis a través del análisis estadístico de los frutos obtenidos. El costo de implementar un sistema de riego inteligente diseñado y ensamblado de forma individual y propia es muchísimo menor que los sistemas de riego que existen actualmente en el mercado. Lavoa (2016); afima que el modelo matemático de evapotranspiracion basado en el metodo de García y López, Penman-Monteith y tanque evaporímetro presentaron resultados de evapotranspiración de referencia más reales de acuerdo a las variables climatológicas pero que puede variar de acuerdo a la zona es porque los métodos descritos requieren en muchos casos acceso a las variables climatológicas. Castro, Chamorro, \& Viteri (2015); afirman que un diseño WSN (Wireless Sensor Network) permite capturar los datos de humedad volumétrica en un cultivo de fresas y controlar de forma general o sitio-específica el riego por método por goteo en el cultivo. En base al prototipo desarrollado, el cual hizo posible verificar la existencia de un grado de variabilidad espacial de humedad de suelo cultivable. De esta forma, se evidencia la importancia del uso de herramientas tecnológicas para lograr una caracterización de humedad de suelo promedio mucho más precisa, definir bajo criterios técnicos cuánto y cuándo regar en diferentes áreas del cultivo. Váscones \& Chamba (2013); sustentan, que su sistema de riego GSM funciona de manera eficiente, pero con retardos de 45 segundos, su cobertura se ve afectada de acuerdo al operador móvil que esté trabajando. El uso de módulos XBee para la comunicación inalámbrica presenta pérdidas de señal cuando no se encuentran en línea de vista, lo cual disminuye su alcance y eficiencia.

Para Flores (2015), la utilización de un software basado en el control difuso optimizo el uso del agua para el riego haciendo pruebas pre y post test, también disminuyó el deterioro del cerco del terreno en relación al riego sin software. Mayhua, Ludeña, Tamayo, Cuba, Nuñez, Gonzales \& Lozada (2016); concluyen que con el uso de la implementación de sensores para determinar la humedad en el suelo y la sectorización del terreno para la apertura y cierre de válvulas para el riego según el nivel de 
humedad del suelo se consigue un ahorro de hasta $25.62 \%$ en el consumo de agua por hectárea. El aumento de producción por hectárea ascendió $4.31 \%$, sus resultados han mostrado una mejora en la productividad y un menor consumo de agua por cada hectárea de cultivo. Anaya, Peluffo, Rios, Castro, Carvaja \& Espinosa (2017); llegan a la conclusión que en la agricultura es necesario el desarrollo de herramientas como los sistemas automatizados de riego para el eficiente uso del agua, pues como es evidente en la actualidad donde un país pasa por una época de verano intensa, las pérdidas por la escasez del vital líquido son inmensas en el sector agrícola. Aunque en el mercado se encuentren sistemas autónomos y automatizados, la gran mayoría de ellos son difíciles de acceder debido al elevado costo económico que presentan, por esto se hace necesario el desarrollo de sistemas similares tratando de disminuir sustancialmente el costo para ponerlo a disposición de la sociedad.

Ravazzani, Corbari, Morella, Gionoli \& Mancini (2012), sostienen basado en el uso del método de Hargreaves- Samani y FAO-56 PM para estimar la evotranspiración por un periodo de 8 años. La ecuaciónFAO- 56 PM se recomienda como el estándar para calcular ETO (evapotranspiración del cultivo de referencia), pero su aplicación está limitada por la disponibilidad de los datos meteorológicos requeridos. En estas circunstancias, la ecuación de HS (Hargreaves-Samani) sobre la base de la temperatura máxima y mínima del aire se considera una buena alternativa, pero a menudo se requiere el ajuste de los coeficientes empíricos a las condiciones climáticas locales, manteniendo a la FAO-56 PM como punto de referencia. Los resultados muestran una sobreestimación general en la elevación más baja y una subestimación en las estaciones más ventosas en la elevación más alta. Dukes, Shedd, \& Davis (2014); concluyen que los controladores evapotranspiración (ET) pueden ser herramientas muy útiles para mejorar la aplicación de agua de riego porque permiten al propietario "configurarlo y olvidarlo". La mayoría de estos controladores de ET calculan los tiempos y ciclos de riego en función de las entradas de da el usuario y las condiciones climáticas. Sin embargo, estos controladores no pueden optimizar un sistema de riego mal diseñado o mal mantenido. Por ellos los autores recomiendan que el sistema de riego se inspeccione regularmente y que se haga un mantenimiento preventivo. También comentan que los diversos controladores ET disponibles comercialmente tienen diferentes términos de programación, entradas y procedimientos; No existe un modelo estándar. Los fabricantes diseñan los controladores para que los instalen contratistas expertos que entiendan las distintas entradas. La programación del controlador para cada paisaje único es crítica para lograr reducir el uso de agua.

Remédios, Arsenio \& Sales (2015); afirma que el desarrollo de un sistema de riego inteligente puede beneficiarse enormemente del conocimiento de la dinámica del suelo y del agua. Y que los factores que influyen en la optimización del ahorro de agua, la estimación de diferentes enfermedades en el cultivo mediante técnicas de aprendizaje automático (machine learning) o determinar que cultivo es apropiado según las condiciones del suelo. Hongal et al. (2017) afirman que Internet de las cosas (IOT) desempeña un papel crucial en la agricultura inteligente. La agricultura inteligente es un concepto emergente, porque los sensores de IoT son capaces de proporcionar información sobre sus campos agrícolas loT y la agricultura inteligente utilizando la automatización. El uso de loT puede proveer un aumento en la producción a bajo costo al monitorear la eficiencia del suelo, la temperatura y la humedad, el monitoreo de la lluvia, medir eficiencia de los fertilizantes, monitorear la capacidad de almacenamiento de los tanques de agua y la detección de robos en áreas agrícolas. La combinación de métodos tradicionales con las últimas tecnologías como Internet de las cosas y una red de sensores inalámbrica (Wireless Network Sensor) puede llevar a la modernización agrícola. Una red de sensores inalámbricos puede recopilar a los datos de diferentes tipos de sensores y los envía al servidor principal mediante un protocolo en específico.

Nikesh Gondchawar (2015) propuso un trabajo sobre la agricultura inteligente basada en loT. El objetivo de su trabajo fue hacer que la agricultura sea inteligente utilizando la automatización y las tecnologías sobre Internet de las Cosas. Por ello desarrollo un robot controlado a distancia basado en GPS, el cual realizaba las operaciones como desherbar, rociar, detectar la humedad, etc. También su trabajo incluye irrigación inteligente con control inteligente y toma de decisiones inteligente basada en datos de campo para realizar una gestión inteligente de almacenes. Todas las operaciones fueron controladas por un dispositivo inteligente y se realizaron mediante la interconexión de sensores a 
través módulos ZigBee, cámaras y actuadores con un controlador Raspberry Pi. En su proyecto logro conectar los sensores y microcontroladores exitosamente con tres Nodos que utilizan un Raspberry Pi y la comunicación inalámbrica. Mat, Rawidean, Nizar \& Mat (2018) sostienen que con la agricultura inteligente se puede construir campos que sean monitoreados con ayuda de sensores así se forma una red inalámbrica para lograr un sistema de irrigación inteligente. En su caso lograron un sistema baso en solo un sensor de humedad incrementar el rendimiento del agua en el riego.

De ahí las interrogantes ¿Cuál es la efectividad de la implementación de un sistema de Internet de las Cosas para optimizar la Gestión del agua en la Agricultura de la Región Tacna?, ¿Cómo optimiza la aplicación de un sistema de Internet de las Cosas en la agricultura de la Región Tacna? y ¿Cuán efectiva la implementación de un sistema de Internet de las Cosas en la solución del problema propuesto?

La presente investigación es importante porque permite al agricultor de la región Tacna tener un sistema capaz de funcionar de manera autónoma, recolecta información del microclima en el ambiente, ahorro energético y principalmente optimizar el recurso hídrico que es muy apreciado en una región que potencialmente sufrirá una escasez de agua a mediano y largo plazo sino se toman medidas para remediarlo. La presente investigación aporta un diseño a bajo costo de un sistema integrado inteligente basado en una aplicación de Internet of Things, integrando una app web, dispositivo embebido y cloud computing. Teniendo una infraestructura como servicios (IaaS) en Amazon Web Services.

De acuerdo a un reporte de la Organización de las Naciones Unidas ([FAO],2013), ya es un hecho que la industria agrícola será más importante que nunca en las próximas décadas. El mundo necesitará producir un 70\% más de alimentos para el 2050 que en el 2006 para alimentar a una creciente población en el mundo. Para logar satisfacer esa demanda, los agricultores, las empresas agrícolas y Start-ups están recurriendo a Internet de las cosas para la recolección y el análisis de datos para lograr una mayor capacidad de producción. El uso de Internet de las cosas (loT, Internet of Things) garantiza un impulso el futuro de la agricultura al siguiente nivel. La agricultura inteligente o Smart farming ya se está convirtiendo en algo más común entre los consumidores lejanos, por ello la agricultura que usa tecnologías actuales se está convirtiendo rápidamente en un estándar gracias a la aplicación de drones, robots y sensores.

\section{Objetivo}

Demostrar la efectividad de la implementación de un sistema de Internet de las Cosas en la optimización de la gestión del agua en la agricultura de la Región Tacna, 2018.

\section{Metodología}

Un estudio patrocinado por las Naciones Unidas y el Banco Mundial indica que la agricultura deberá proporcionar hasta el 70\% del aumento para cubrir las necesidades alimentarias del mundo en 2025. Postel (1999) indica que los niveles de producción de alimentos necesarios en 2025 podrían requerir hasta 2,000 kilómetros cúbicos (1,600 millones de acres-pies) de agua adicional para riego del cultivo. La gestión del agua y los rendimientos de los cultivos pueden mejorarse mediante el uso incrementado de métodos confiables para estimar la evapotranspiración de los cultivos (ET), riego de precisión avanzado en conjunto con los métodos de precesión como riego por goteo o expansión.

Cabe mencionar que una buena gestión del agua en la agricultura también incluye la gestión del agua utilizada en la producción, producción de la pesca continental y producción ganadera, y la mejora de la gestión en estas áreas de producción es la respuesta a la seguridad alimentaria en el mundo.

El tipo de investigación es aplicada, ya que soluciona problemas prácticos, buscando nuevos 
conocimientos para poder ser aplicados en el campo de la ingeniería. Esta investigación tiene como fin demostrar la efectividad de la aplicación de un sistema de internet de las cosas como método para aumentar la efectividad del uso agua en la irrigación. Catorce parcelas del asentamiento 4. Utilizan el pozo de agua 106 del sector agrario de la Yarada- Los Palos. Se cuenta con una parcela con un área de cultivo de Olivo de aproximadamente $3535 \mathrm{~m}^{2}$, con un reservorio de 600000 litros de agua aproximadamente.

Variable: Sistema de Internet de las Cosas. Conjunto de elementos relaciones entre sí para realizar uno o varios procesos en específico, procedimientos, reglas, datos e información asociada a estas que funcionan específicamente en diferentes dispositivos. En referencia a lo mencionado anteriormente entonces definiremos que un sistema de Internet of Things que serán instrucciones lógicas capaces de interactuar con dispositivos, sensores, actuadores desde internet y en tiempo real, procedimientos relacionados a la seguridad, documentación, datos e información asociados al control y monitoreo de metadatos obtenido por sensores.

Variable: optimizar la gestión del agua en la agricultura se entiende como optimizar al proceso de modificar un sistema para mejorar su eficiencia y el uso de los recursos disponibles. Usaremos tecnologías para optimizar el proceso del regadío de los cultivos en la agricultura. Llegar a automatizar el proceso con un dispositivo inteligente, monitorear el cultivo desde una aplicación web y centralizar diferentes dispositivos interconectados entre sí a través un servidor remoto alojado en la nube.

Instrumentos: Uso del Sistema de Internet de las Cosas denominado Damla para evaluar la efectividad del riego basándonos en los datos capturados por: Sensor de Radiación UV, Sensor de humedad de suelo, Sensor de humedad del aire, Sensor de temperatura ambiente.

Los datos son procesados para determinar el tiempo efectivo de riego en el cultivo.

La recolección de datos mediante en encuestas es solo para saber si existe la necesidad de un sistema de internet de las cosas para optimizar el agua. Los datos recolectados por el dispositivo desarrollado, Damla, nos ayuda a saber los intervalos de tiempo de riego del cultivo.

La presentación de la información a través de tablas luego de procesamiento de esta, por lo tanto, se hará una presentación tabular para la discusión de los procedimientos lógicos de la deducción e inducción al comparar los resultados con el sistema Damla y el sistema de riego tradicional por controlador de tiempo de riego.

\section{Resultados}

Según se observa en la tabla, con respecto al funcionamiento del proceso actual de irrigación en la zona de la Yarada- Los Palos, asentamiento 4, los lugareños, en una muestra de 30 personas, afirman que no se hace uso de Sistemas de Internet de las Cosas

con respecto al conocimiento actual, ni una persona sabe con profundidad sobre el tema. Un $15 \%$ tiene un conocimiento superficial. El $20 \%$ escucho el término, pero no investigó. Y como apreciamos, la mayoría de ellos, el $70 \%$, no conoce nada sobre el tema.

Con respecto a la utilidad de la integración de Internet de las Cosas en nuestras vidas, se puede apreciar que un $100 \%$ de los encuentras, reconoce la importancia de la implementación de un sistema de Internet de las Cosas en nuestras vidas. 
Tabla 1

Uso conocimiento y utilidad del internet de las cosas en la agricultura en la Región de Tacna

\begin{tabular}{|c|c|c|}
\hline & $\mathbf{n}$ & $\%$ \\
\hline \multicolumn{3}{|c|}{ ¿Se hace uso de Sistemas con internet de las cosas en la agricultura en la Región Tacna } \\
\hline $\mathrm{Si}$ & 0 & 0,00 \\
\hline No & 30 & 100,00 \\
\hline Total & 30 & 100,00 \\
\hline \multicolumn{3}{|c|}{ ¿Cuál es el conocimiento actual que se posee sobre sistemas de Internet de las Cosas? } \\
\hline Conozco el tema a profundidad. & 0 & 0,00 \\
\hline Sé algo del tema. & 4 & 13,33 \\
\hline $\begin{array}{l}\text { Conozco el término, pero no he } \\
\text { investigado sobre el tema }\end{array}$ & 5 & 16,67 \\
\hline No conozco nada sobre domótica & 21 & 70 \\
\hline Total & 30 & 100 \\
\hline \multicolumn{3}{|c|}{ ¿Cree que es útil la integración de Internet de las Cosas en nuestras vidas? } \\
\hline Si & 30 & 100,00 \\
\hline No & 0 & 0,00 \\
\hline Total & 30 & 100,00 \\
\hline
\end{tabular}

Tabla 2

Gestión de agua en la agricultura por el nivel, esfuerzo y gasto

\begin{tabular}{llll}
\hline & Frecuencia & \% & \%Acumulado \\
\hline \multicolumn{4}{c}{ Nivel } \\
\hline Alto & 4 & 13,33 & 13,33 \\
Medio & 15 & 50,00 & 63,33 \\
Bajo & 11 & 36,67 & 100,00 \\
Total & 30 & 100,00 & \\
\hline \multicolumn{4}{c}{ Esfuerzo } \\
\hline Alto & 0 & 0,00 & 0,00 \\
Medio & 12 & 40,00 & 40,00 \\
Bajo & 18 & 60,00 & 100,00 \\
Total & 30 & 100,00 & \\
\hline \multicolumn{4}{c}{ Gasto } \\
\hline Alto & 18 & 60,00 & 60,00 \\
Medio & 6 & 20,00 & 80,00 \\
Bajo & 6 & 20,00 & 100,00 \\
Total & 30 & 100,00 & \\
\hline
\end{tabular}

A la pregunta ¿Cómo consideraría el nivel de la gestión del agua en la agricultura de la región Tacna?, los resultados muestran que, con respecto a la gestión del agua en la agricultura, un $50 \%$ considera que existe un nivel medio de la gestión del agua, un $36 \%$ considera que el nivel de gestión es bajo, mientras que una pequeña minoría piensa que es alto.

A la pregunta ¿Cuál es el esfuerzo que se realiza en la actualidad para mejorar la gestión agua en la agricultura de la región Tacna?, con respecto a la gestión del agua en la agricultura, nadie indicó que se hiciera un gran esfuerzo para mejorar la gestión del agua, mientras que el $40 \%$ considera que se hace un esfuerzo mediano y un $60 \%$ considera que el esfuerzo bajo. Por lo tanto, podemos afirmar que los agricultores afirman que existe un nivel bajo de esfuerzo en la gestión de agua.

Pregunta ¿Cómo consideras los gastos realizados en la gestión del agua en la agricultura?

Con respecto al gasto realizado en la gestión del agua, que un $60 \%$ considera que gastan mucho en el pago del agua, un $20 \%$ piensa que es medio, así como también las personas que piensan que es bajo 
el costo para la gestión del agua. Por lo tanto, podemos afirmar que existe una cantidad elevada en los gatos realizados para la gestión de agua.

En primer lugar, el objetivo de las encuestas fue determinar si hay una necesidad entre los agricultores de mejorar su proceso y/o método de riego para optimizar el uso del agua en el cultivo. Entonces, se determinó desde el principio de la presente investigación que el problema general se basa en la efectividad de implementar un sistema de Internet de las Cosas para optimizar la gestión del agua en la agricultura basándonos en que existe un escaso recurso hídrico en nuestra región.

La red de riego está compuesta por un reservorio con $600000 \mathrm{I}$. El sistema de riego se encuentra en un cuarto de control, donde se encuentran los filtros y una bomba centrifuga de caudal de $5 \mathrm{HP}$, con un caudal de $350 \mathrm{l} / \mathrm{min}$. Funcionamiento nominal del sistema de riego, tienen como entrada y salida una tubería de 2 ", una troncal que distribuye el agua de 2 ". Aproximadamente 40 tubos de $1 / 2{ }^{\prime \prime}$, los cuales están conectados a la trocal, para luego distribuir el agua por el método de goteo a más de 80 ejemplares de olivo. Los motores son activados por un controlador marca Galcon, el cual es programado para que a ciertas horas se riegue el cultivo, sin alguna retroalimentación acerca del clima.

Para obtener un promedio de horas riego durante el transcurso del año, primero se revisó el controlador utilizado por los dueños de área de cultivo de olivo, para tratar de extraer sus datos de la EEPROM del controlador, pero no se pudo ya que se pudo haber perdido el programa interno. Por ello se estimó el tiempo con una comunicación directa con el agricultor para estimar la cantidad de agua consumida.

En el siguiente cuadro demuestra la cantidad de horas irrigadas por mes.

\section{Figura 1}

Duración de regadío (Horas)

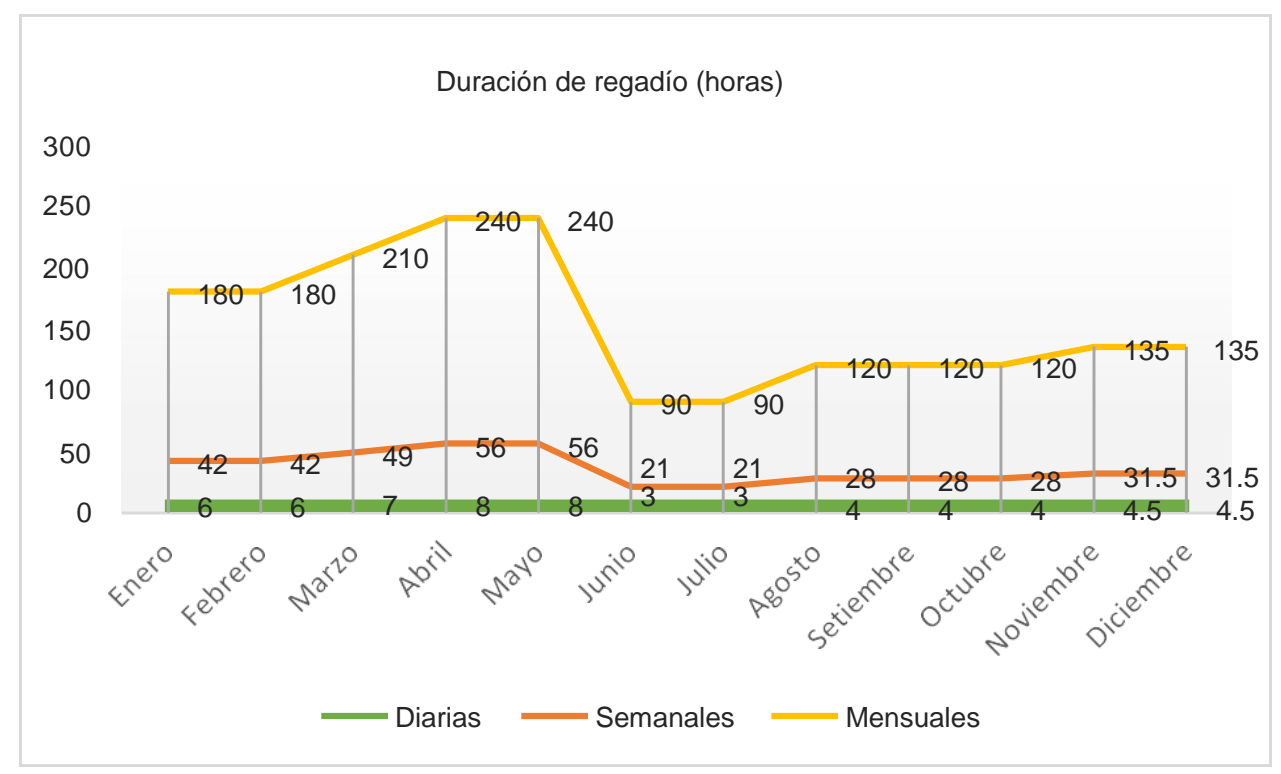

En la figura 1 muestra la cantidad de horas de riego efectuadas en diferentes meses, segmentadas entre diaria, semanales y mensuales. Los cuales muestran que en estaciones de verano es cuando se incrementa las horas de riego porque las altas temperaturas evaporan en agua del cultivo.

A continuación, se determinó la eficiencia del transporte de agua, es decir cuan eficiente es el sistema de tuberías para el riego. Se determinó la eficiencia del transporte $90.2 \%$ 
Los datos analizados están basados en el tiempo en que Damla activa las bombas de agua para regar el cultivo, estos datos sirven para determinar un aproximado de cuánta agua consumo en relación al tiempo en que las bombas de agua riegan el cultivo. Los datos capturados son a partir de la fecha en que Damla empezó a operar.

\section{Figura 2}

\section{Uso del agua con Damla}

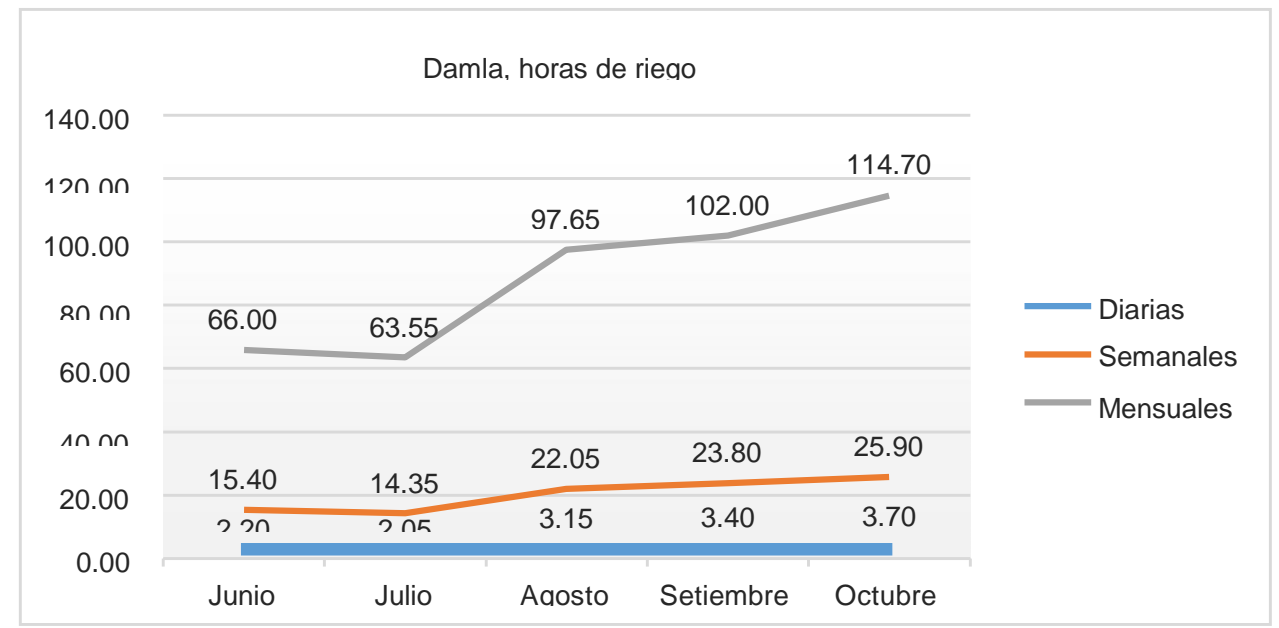

En la figura 2, observamos un promedio de las horas de riego efectuadas entre los meses de junio y octubre. La tendencia a subir las horas de riego también se replica como el sistema de convencional de riego, es decir por la estación o temporada del año.

Tomamos los datos en relación a los meses en que ambos sistemas trabajaron en paralelo, para así determinar el nivel de efectividad que tiene Damla sobre un sistema tradicional de riego.

Figura 3

Eficiencia entre sistemas de riego (Horas)

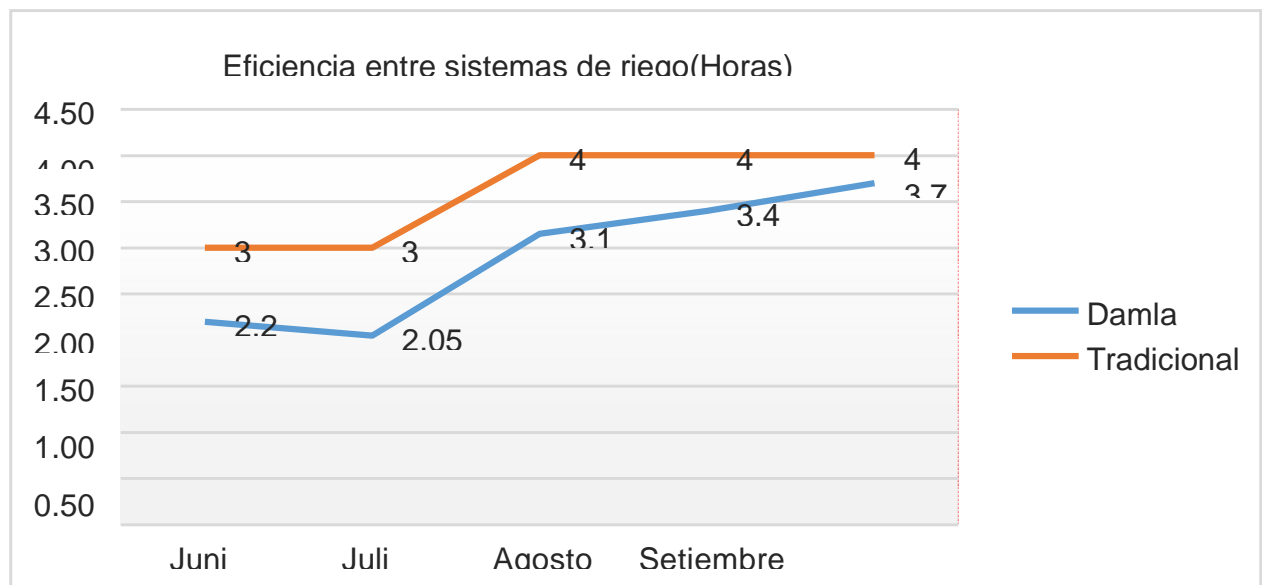

Observamos una tendencia en horas utilizadas en el regadío entre ambos sistemas. Podemos observar que, con el sistema de internet de las cosas, Damla tenemos un menor tiempo de riego, el cual equivale a mayor ahorro de agua. 
Para hacer las pruebas se instaló el dispositivo aproximadamente a unos 50 metros del cuarto del control. Ubicado el lugar debemos cavar un agujero que donde el follaje de la planta no haga sombra con nuevo dispositivo.

El agujero tiene que tener una profundidad entre 30 y $40 \mathrm{~cm}$ Para que el dispositivo Damla Sensores este bien asegurado y firme ante las perturbaciones del medio ambiente.

Como podemos apreciar, el dispositivo está a unos metros de un árbol de olivo, lo importante es que es que el follaje no cubra por completo el dispositivo, así pueda recargar la batería que su optima operación.

Una media de la cantidad de agua utilizada diariamente entre ambos sistemas. Podemos observar que con Damla, obtenemos un ahorro de agua basándonos en el tiempo de riego. Entonces, tomando los resultados de las horas diarias podemos determinar la mediana de las horas. Es decir que Damla es $\mathbf{2 1 . 2 5 \%}$ más efectivo que el sistema tradicional de goteo controlado con controladores de tiempo.

Teniendo en cuenta la media entre ambos sistemas, podemos decir que: Damla ahorra diariamente 17849 litros de agua.

Costo Beneficio, actualmente existe una empresa reconocida en la región Tacna, Silpay, el cual fue quien instaló el sistema de riego en área de cultivo de esta investigación. El cual tuvo un costo total de $\mathrm{S} / 32,000.00$, treinta y dos mil soles. Tal proyecto de instalación tuvo un funcionamiento óptimo los primeros meses y luego el sistema de riego por temporizador empezó a fallar, hasta que el dueño de la parcela volvió a un sistema de riego manualmente.

La propuesta de acuerdo al requerimiento del dueño de la parcela es de proporcionar una solución integral. En ellos se establecieron los siguientes costos.

Se presentó el costo de la optimización total que tendría que pagar el dueño de la parcela para mejorar un área total de 2.4 Hectáreas a solicitud de él. Sin embargo, pasado el año el dueño de la parcela tendrá que pagar una membresía de $\mathrm{S} / .34$ soles para poder monitorear y controlar el sistema de riego.

Los beneficios que obtendría es una:

- Automatización de riego del cultivo.

- Ahorro de agua de hasta un $21.25 \%$.

- Ahorro energético relación directa al uso de las bombas eléctricas para el riego.

- Ahorro de tiempo.

- Una plataforma para que pueda monitorear el cultivo.

Los gastos mencionados en los anexos son referentes a un área menor al área total de dicha parcela.

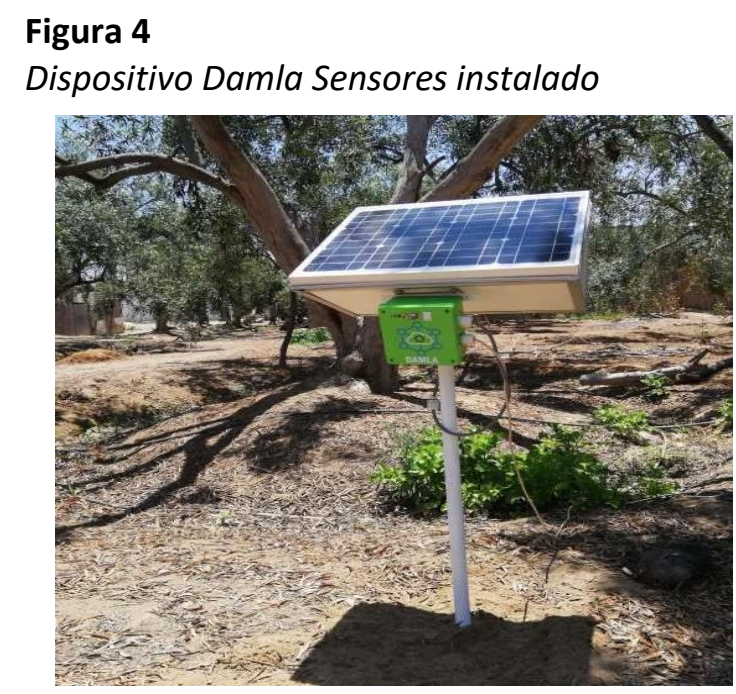


Tabla 3

Costo de la optimización requerida

\begin{tabular}{|c|c|c|c|c|}
\hline Ítems & Unidades & Precio(S/.) & Subtotal(S/.) & \\
\hline Dispositivo Damla Acuador & 1 & 171 & 171 & \\
\hline Dispositivo Damla Sensores & 4 & 450 & 1800 & \\
\hline Router CPE Wifi & 1 & 400 & 400 & \\
\hline Antena Bidireccional & 1 & 320 & 320 & \\
\hline \multicolumn{5}{|l|}{$360^{\circ}$} \\
\hline Válvulas Solenoide 2" & 4 & 173.68 & 694.72 & \\
\hline \multirow[t]{2}{*}{ Acoples } & 8 & 12 & 96 & \\
\hline & & Total & 3481.72 & 3481.72 \\
\hline \multicolumn{5}{|l|}{ Gastos Profesionales } \\
\hline Instalación & 1 día & 600 & 600 & \\
\hline \multirow[t]{2}{*}{ Membresía mensual } & 1 año & 34 & 408 & \\
\hline & & Total & 1008 & 1008 \\
\hline
\end{tabular}

\section{Discusión}

El resultado del procesamiento de la información con Damla, ha permitido obtener resultados positivos para la optimización del ahorro de agua en la irrigación del cultivo, pero es importante mencionar que el proyecto podría funcionar de mejor manera en invernaderos, ya que se aprovecharía mejor sus sensores como el de temperatura y humedad, las cuales en un invernadero servirían como elemento de control para cambiar la temperatura del ambiente y la humedad, así poder tener mejores resultados en cuanto la calidad del cultivo.

Si bien existen diferentes métodos para la mejora de riego en el cultivo, por ejemplo, determinar la evapotranspiración con el método de FAO, Penman y Penman-Monteith o HargreavesSamani los cuales determinan la evapotranspiración para que luego se pueda hacer un cronograma de riego, pero en la presente investigación se utilizó una función hibrida para que el sistema pueda tomar decisiones en tiempo real y no necesariamente diseñe un cronograma de riego.

Las propuestas para mejorar el uso del agua en la Región de Tacna son escasas de innovación tecnología y deficientes. Por ello es de mucha importancia que el proyecto Damla pueda seguir implementándose en diferentes campos de cultivo. Por ello se tendrá que elaborar un artículo científico para su presentación en el colegio de Ingeniero de Ingenieros del Perú, ANA y demás organizaciones de interés.

También Internet de las Cosas es un mercado emergente y con la llegadade la red $5 \mathrm{G}$ aún más. Es decir, al diseñar un dispositivo electrónico, el proyecto fácilmente puede escalar para seguir conectando otros dispositivos a internet el cual vuelve al proyecto realizado en mi investigación en un potencial producto y/o servicio comercializable que principalmente esté al alcance de los agricultores.

\section{Conclusiones}

Con una reducción de $21.25 \%$ de tiempo el riego efectivo del cultivo, se concluye que un 
sistema de Internet de las Cosas optimiza la gestión del agua en la agricultura ya que las horas de riego está directamente relacionada al agua utilizada para el riego.

Se evidencia notables mejoras respecto a otros métodos tradicionales asegurando un mejor uso de un recurso natural tan escaso como el agua. Pero también es importante resaltar que el sistema provee un ahorro energético, ya que está directamente relacionado al uso de las bombas eléctricas en el riego.

El uso de sensores en un dispositivo electrónico, como Damla, proporciona mejoras en el proceso de riego en la Agricultura. Es decir, profesionales relacionados a la tecnología e información pueden cubrir soluciones relaciones sobre un mercado emergente de internet de las cosas.

En las estaciones como la primera o verano, es cuando más se requiere del agua, llegando a escasear el agua en los reservorios el cual provoca que a veces se pierda la cosecha de la aceituna por la falta de riego en el cultivo. Sin embargo, esta solución está basada en la nueva revolución industrial 4.0, Internet de las cosas y tecnologías en la nube. Ahora los agricultorespueden usar menos agua para su cultivo, tener una herramienta tecnológica de ingeniería robusta y de primer mundo que permite automatizar, controlar y monitorear su cultivo desde cualquier parte de mundo

\section{Referencias Bibliográficas}

Ahamed, T. (2019). Deep learning and loT-based pump systems for precision irrigation. Tokyo: apotokyo.

Alegria, J. F. (2013). The Challenges of water resources management in Peru.Lima.

Anaya, A., Peluffo, D., Rios, J., Castro, J., Carvajal, D., \& Espinosa, L. (2017).

Sistema de Riego Basado en la Internet de las Cosas. San Miguel de Ibarra.

Bennett, S. (1993). A History of Control Engineering, 1930-1955. En S. Bennett, A History of Control Engineering, 1930-1955 (pág. 250). Peter Peregrinus Ltd.

Castro, N., Chamorro, L., \& VIteri, C. (2015). Una red de sensores inalámbricos para la automatización y control del riego localizado. Pasto.

Ciencias. (22 de enero de 2018). www.elcomercio.pe. Obtenido de https://elcomercio.pe/tecnologia/ciencias/razones-escasez-agua-mundo- noticia-490577

Conor, A. (2012). Water resource management, group theory and the tragedy of the commons in Tacna, Peru. Toronto.

Davis Internacional. (1 de junio de 2018). JUPM. Obtenido de http://www.jupm.pe: http://www.jupm.pe/desarrollo/notas/15278819684/busqueda/fecha/2018-06- 25

Dias Sales, N. F. (2015). Cloud-based Wireless Sensor and Actuator System for Smart. Lisboa.

Dukes, M., Shedd, M., \& Davis, S. (2014). Smart Irrigation Controllers: Operation of EvapotranspirationBased Controllers. Florida: Electronic Data Information. Factory Fresh. (9 de Junio de 2016). Technology Quarterly. Obtenido de economist: https://www.economist.com/technologyquarterly/2016-06-09/factory-fresh

Flores Aguilar, D. I. (2015). Software de riego inteligente basado en control difuso para mejorar la administración del consumo de agua en los campos del Valle Chicama. Trujillo.

Fry, A. (2005). Agua: Hechos y tendencias. Texas: Earthprint Ltd. Guzman, J. (2013). Arduino. Madrid: MediaLab.

Hongal, A., M, J., \& S, P. (2017). Agriculture, lot baed monitoring system in Smart Agriculture. Karnataka: IEEE.

José, M., Calero, V., \& Marín, I. (2013). Diseño e Implementación de un Sistema de Riego Inteligente basado en Sensores y Módulos de Radiofrecuencia para Transmisión y Sistema de Control. Guayaquil.

Lavoa Pastrana, S. (2016). Medición y modelamiento de la evapotranspiración real del cultivo de clavel en invernadero en La Sabana de Bogotá, usando lisímetros electrónicos de pesada. Bogota. 
Luquet, D., Vidal, A., Smith, M., \& Dauzat, J. (2005). 'More crop per drop': how to make it acceptable for farmers? Montpellier: ELSEVIER.

Mat, I., Rawidean, M., Nizar, A., \& Mat, I. (2018). Smart Agriculture Using Internet of. Malasia: IEEE.

Mayhua, E., Ludeña, J., Tomayo, J. C., Nuñez, Á., Gonzales, N., \& Lozada, D. (2016). Sistema de riego por goteo automático utilizando una red de sensores inalámbricos. Arequipa.

Ravazzani, G., Corbari, C., Morella, S., Gionoli, P., \& Mancini, M. (2012). Modified Hargreaves-Samani Equation for the Assessment of Reference Evapotranspiration in Alpine River Basins. Journal of Irrigation and Drainage Engineering.

Remédios, O., Arsenio, A., \& Sales, N. (2015). Wireless Sensor and Actuator System for Smart Irrigation on the Cloud. Milan: IEEE.

Sekharm, R., Manujunatha, S., \& Sundeep, K. (2013). Evapotranpiration Model Using Al Controller for automatic. Bangalore: International Journal of Computer Trends and Technology.

Sentlinger, K. (12-de Agosto de 2004). The water Project. Obtenido de the water Project: https://thewaterproject.org/water-scarcity/water-scarcity-and- agriculture

Trank.in. (2 de mayo de 2017). How loT's are changing the Fundamentals of "Retailing". Obtenido de trank: http://trak.in/tags/business/2016/08/30/internet-of-things-iot-changingfundamentals-of-retailing/

Váscones Cuzco, J. C., \& Chamba Tenemaza, F. d. (2013). Diseño e implementación de un sistema de riego automatizado y controlado de forma inalámbrica para una finca ubicada en el sector popular de Balario. Guayaquil.

Villabos, F., Orgaz, F., Testi, L., \& Fereres, E. (2000). Measurement and modeling of evapotranspiration of olive. Cordoba: European Journal of Agronomy. 